\title{
Observable effects caused by vacuum pair creation in the field of high-power optical lasers
}

\author{
David B. Blaschke ${ }^{a, b, c}$, Andrey V. Filatov ${ }^{d}$, Irina A. Egorova ${ }^{b, d}$, \\ Alexander V. Prozorkevich ${ }^{d}$ and Stanislav A. Smolyansky ${ }^{d}$ \\ ${ }^{a}$ Institute of Theoretical Physics, University of Wrocław, 50-204 Wrocław, Poland \\ ${ }^{b}$ Bogoliubov Laboratory for Theoretical Physics, JINR Dubna, RU-141980 Dubna, Russia \\ ${ }^{c}$ Institute of Physics, University of Rostock, D-18051 Rostock, Germany \\ ${ }^{d}$ Saratov State University, RU-410026, Saratov, Russia
}

\begin{abstract}
We consider the possibility of an experimental proof of vacuum $e^{+} e^{-}$pair creation in the focus of two counterpropagating optical laser beams with an intensity of the order of $10^{20}-10^{22} \mathrm{~W} / \mathrm{cm}^{2}$. Our approach is based on the collisionless kinetic equation for the distribution function of the $e^{+} e^{-}$pairs with the source term for particle production. As a possible experimental signal of vacuum pair production we consider the refraction of a high-frequency probe laser beam by the produced $e^{+} e^{-}$plasma to be observed by an interference filter. The generation of higher harmonics of the laser frequency in the self-consistent electric field is also investigated.
\end{abstract}

Keywords: vacuum pair creation, strong laser field, refraction index

\section{INTRODUCTION}

The great progress in laser technology expected in near future ${ }^{1,2}$ raises hopes for a sought-after experimental proof of some non-linear effects in quantum electrodynamics, in particular, the dynamical Schwinger effect of vacuum pair creation predicted a long time ago. ${ }^{3-5}$

As is known ${ }^{5}$ no pairs can be created when both invariants of the electromagnetic field vanish, $\mathbf{E}^{2}-\mathbf{B}^{2}=0$, $\mathbf{E B}=0$. The field produced by focussed laser beams is very close to such a configuration ${ }^{6}$ and therefore the pair creation is expected to be essentially suppressed. On the other hand, it should be possible to avoid the lightlike field configuration with a spatially uniform field created in an antinode of the standing wave produced by the superposition of two coherent, counter-propagating laser beams. ${ }^{7}$ Since the Schwinger effect is non-perturbative and it requires an exact solution of the dynamical equations it is customary to approximate the complicated structure of a real laser field by a spatially uniform time-dependent electric field. According to early estimates ${ }^{6-12}$ the effect of vacuum creation should not be observable with presently available laser parameters. Essentially, these estimations concerned the pairs which remain after the laser pulse when the electric field disappears. Technically, time interval considered has to be an integer multiple of the field period. Another possibility was investigated in the works, ${ }^{13-17}$ where the dynamical pair density during the action of laser pulse changes periodically with twice the field frequency and its mean value exceeds the residual density by several order of magnitude. In that case, plenty of quasiparticle $e^{+} e^{-}$pairs are created even in weak time-dependent fields $\omega^{2} \ll E \ll m^{2} / e$. Though these pairs disappear together with driving field, they can interact in standard ways (scattering, annihilation etc.) during the period of field presence some traces of these processes should be revealed.

The nonlinear effects due to the interaction of intense laser beams with the vacuum can manifest themselves in various physical phenomena, e.g., by nonlinear Thomson scattering, ${ }^{18}$ damping of electromagnetic waves in a plasma due to $e^{+} e^{-}$pair production, ${ }^{12}$ two-photon annihilation, ${ }^{19}$ the non-linear Breit-Wheeler process, ${ }^{20}$ vacuum birefringence ${ }^{21}$ and dichroism. ${ }^{22}$ Some other interesting possibilities are considered recently in Refs. ${ }^{23,24}$ The estimation of the two-photon annihilation rate $^{14}$ shows that the field of an optical laser with intensity $10^{20} \mathrm{~W} / \mathrm{cm}^{2}$ can produce 5-10 annihilation events per laser pulse with irradiation of $\sim 1 \mathrm{MeV} \gamma$-quanta. The corresponding $\gamma$-quanta can be registered outside the focus of the counter-propagating laser beams.

In present work, we consider whether it is possible to observe experimentally a refraction index of the transient $e^{+} e^{-}$plasma created in the laser focus by means of some interference scheme with a high-frequency probe laser. 
In the collision of two counter-propagating laser beams form thin spatial regions with quasi-homogeneous electric field where conditions for $e^{+} e^{-}$pair creation are fulfilled. One of the split beams of the probe laser passes through the plasma region and obtains a phase shift relative to the second beam that can be measured by an interference filter. The density of created pairs oscillates with the double laser frequency. Therefore, a stable interference pattern can be obtained provided the probe laser pulse is short enough. A simple estimate shows that the intensity of state-of-the-art optical lasers is not sufficient for such an experiment but the critical intensity can be reached in near future. The $e^{+} e^{-}$pairs moving in the laser field generate a secondary electric field which contains higher harmonics of the laser frequency, the most intense of which is third one.

\section{MODEL}

We suppose that two counter-propagating laser beams (Fig 1) form a standing-wave field

$$
\begin{aligned}
& \mathbf{E}=\left\{0,0,2 E_{m} \phi(t) \sin \left(\omega_{0} t\right) \cos (k x)\right\}, \\
& \mathbf{B}=\left\{0,2 E_{m} \phi(t) \cos \left(\omega_{0} t\right) \sin (k x), 0\right\},
\end{aligned}
$$

where $\phi(t)$ is some envelope function. The small spatial regions in the vicinity of the anti-nodes of the electric field (Fig. 2) can be considered as areas with quasi-homogeneous electric field

$$
\mathbf{E}_{e x}(t)=\left(0,0, E_{e x}(t)\right), \quad E_{e x}(t)=E_{0} \phi(t) \sin \omega_{0} t,
$$

which are capable of pair creation. The Dirac equations can be solved exactly in such a field ${ }^{25,26}$ and the distribution function of created quasiparticles $f(\mathbf{p}, t)$ can be found at any time as the solution of the following kinetic equation ${ }^{27}$

$$
\begin{aligned}
\frac{d f(\mathbf{p}, t)}{d t}= & \frac{1}{2} \Delta(\mathbf{p}, t) \int_{t_{0}}^{t} d t^{\prime} \Delta\left(\mathbf{p}, t^{\prime}\right)\left[1-2 f\left(\mathbf{p}, t^{\prime}\right)\right] \cos \theta\left(\mathbf{p}, t^{\prime}, t\right), \\
\Delta(\mathbf{p}, t) & =e E(t) \frac{\sqrt{m^{2}+p_{\perp}^{2}}}{\varepsilon^{2}(\mathbf{p}, t)} \\
\varepsilon(\mathbf{p}, t) & =\sqrt{m^{2}+p_{\perp}^{2}+\left[p_{\|}-e A(t)\right]^{2}} \\
\theta\left(\mathbf{p}, t^{\prime}, t\right) & =2 \int_{t^{\prime}}^{t} d t_{1} \varepsilon\left(\mathbf{p}, t_{1}\right),
\end{aligned}
$$

where $e$ and $m$ are the particle charge and mass, $A(t)$ is the vector potential of the electric field. The total field $E(t)$ is defined as the sum of the external (laser) field $E_{e x}$ and the self-consistent internal field $E_{i n}$, which is determined by Maxwell's equation

$$
\begin{aligned}
\frac{d E_{i n}(t)}{d t}=-\frac{e}{(2 \pi)^{3}} \int \frac{d \mathbf{p}}{\varepsilon(\mathbf{p}, t)}\{2 & p_{\|} f(\mathbf{p}, t) \\
& \left.+\sqrt{m^{2}+p_{\perp}^{2}} \int_{t_{0}}^{t} d t_{1} \Delta\left(\mathbf{p}, t_{1}, t\right)\left[1-2 f\left(\mathbf{p}, t_{1}\right)\right] \times \cos \left(2 \int_{t_{1}}^{t} d t_{2} \varepsilon\left(\mathbf{p}, t_{2}, t\right)\right)\right\}
\end{aligned}
$$

The total current density on the r.h.s. of Eq. (7) is the sum of the conductivity and vacuum polarization contributions, respectively.

The equation (3) was investigated carefully with reference to the conditions in heavy ion collisions ${ }^{29}$ and in laser fields. ${ }^{14,30,31}$ As opposed to the first case, where the characteristic times for pair production $\tau_{p} \sim 1 / m$ and the field action $\tau_{f}$ are of the same order, the laser field case corresponds to a situation when these time scales differ by many orders of magnitude. For example, $\tau_{f} / \tau_{p} \sim 10^{8}$ for optical lasers and $\sim 10^{4}$ for X-ray free electron lasers. ${ }^{32}$ As a consequence, the numerical task becomes extremely robust. 


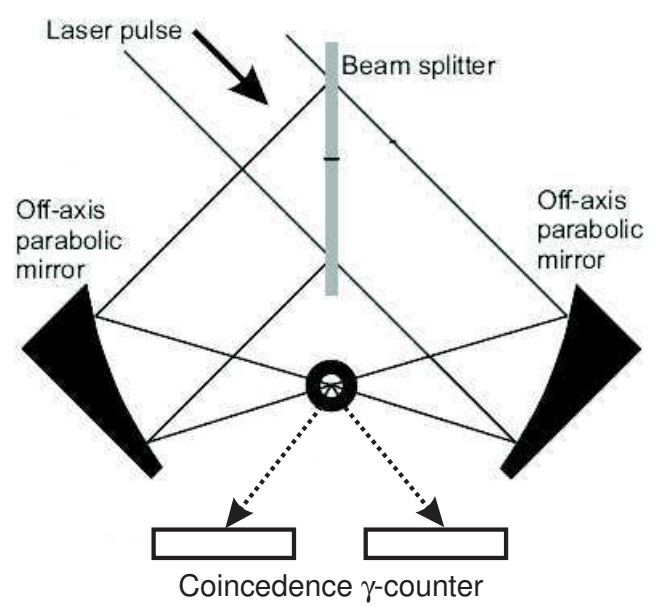

Figure 1. Schematic representation of the experimental setup for two colliding laser pulses. ${ }^{28}$ The main pulse of power laser is divided into two pulses of equal energy by a beam splitter, both pulses are focused in one point by a $45^{\circ}$ parabolic mirror.

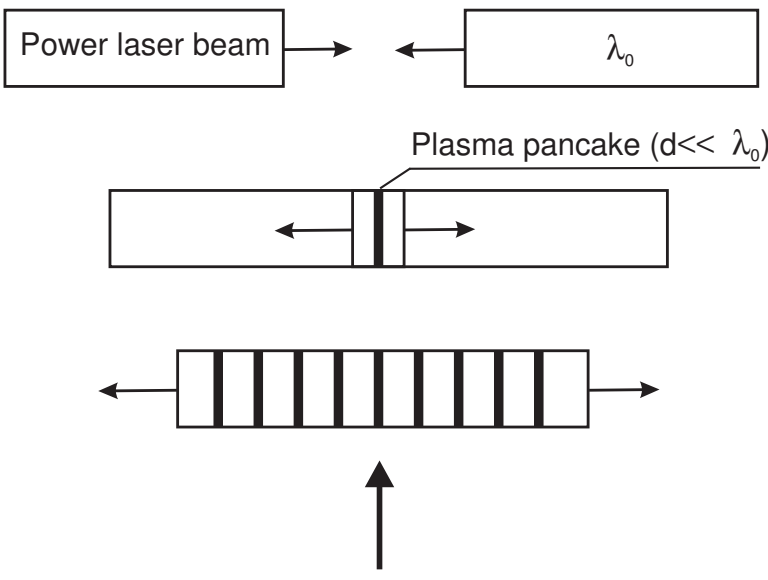

Probe laser beam

$\lambda_{\mathrm{p}}<<\lambda_{0}$

Figure 2. Formation of thin plasma regions ("pancakes") in the collision of two counter-propagating laser beams with wavelength $\lambda_{0}$. The beam of the probe laser has a short wavelength $\lambda_{p} \ll \lambda_{0}$ and propagates perpendicular to the direction of the high-power laser beams.

For the numerical investigation, equation (3) is transformed to a set of linear differential equations ${ }^{27}$

$$
\frac{d f}{d t}=\frac{1}{2} \Delta u, \quad \frac{d u}{d t}=\Delta\left(1-2 f_{a}\right)-2 \varepsilon v, \quad \frac{d v}{d t}=2 \varepsilon u,
$$

where $u, v$ are real auxiliary functions. In such terms, the Maxwell equation (7) becomes

$$
\frac{d E_{i n}(t)}{d t}=-\frac{e}{(2 \pi)^{3}} \int \frac{d \mathbf{p}}{\varepsilon(\mathbf{p}, t)}\left\{2 p_{\|} f(\mathbf{p}, t)+\sqrt{m^{2}+p_{\perp}^{2}} u\right\} .
$$

The system (8) is integrated by the Runge-Kutta method with the initial conditions $f\left(\mathbf{p}, t_{0}\right)=u\left(\mathbf{p}, t_{0}\right)=$ $v\left(\mathbf{p}, t_{0}\right)=0$. The envelope function in (2) is chosen as $\phi(t)=\theta(t)-\theta(t-\tau)$, where $\tau$ is the laser pulse duration. The solution of (3) is used to calculate the dynamical number density

$$
n(t)=2 \int \frac{d \mathbf{p}}{(2 \pi)^{3}} f(\mathbf{p}, t)
$$

and the annihilation rate in unit volume

$$
\frac{d N_{a}}{d V d t}=\int \frac{d \mathbf{p}_{1}}{(2 \pi)^{3}} \frac{d \mathbf{p}_{2}}{(2 \pi)^{3}} \sigma\left(\mathbf{p}_{1}, \mathbf{p}_{2}\right) f_{a}\left(\mathbf{p}_{1}, t\right) f_{a}\left(\mathbf{p}_{2}, t\right) \times \sqrt{\left(\mathbf{v}_{1}-\mathbf{v}_{2}\right)^{2}-\left|\mathbf{v}_{1} \times \mathbf{v}_{2}\right|^{2}},
$$

where $\mathbf{v}$ is the particle velocity and $\sigma$ the annihilation cross-section. The corresponding expression for spinor electrodynamics is ${ }^{19}$

$$
\sigma_{e}\left(\mathbf{p}_{1}, \mathbf{p}_{2}\right)=\frac{\pi e^{4}}{2 m^{2} \tau^{2}(\tau-1)}\left[\left(\tau^{2}+\tau-1 / 2\right) \times \ln \left\{\frac{\sqrt{\tau}+\sqrt{\tau-1}}{\sqrt{\tau}-\sqrt{\tau-1}}\right\}-(\tau+1) \sqrt{\tau(\tau-1)}\right] .
$$

The t-channel kinematic invariant $\tau$ is given by

$$
\tau=\frac{\left(p_{1}+p_{2}\right)^{2}}{4 m^{2}}=\frac{1}{4 m^{2}}\left[\left(\varepsilon_{1}+\varepsilon_{2}\right)^{2}-\left(\mathbf{p}_{1}+\mathbf{p}_{2}\right)^{2}\right] .
$$




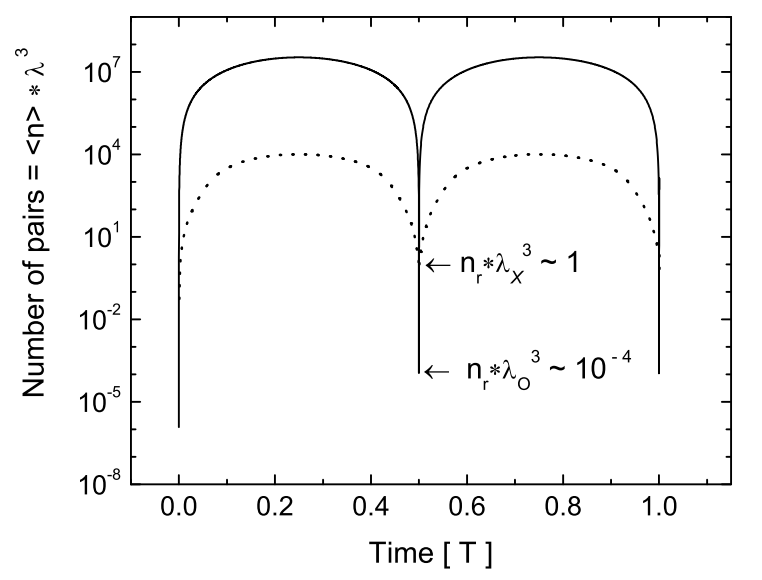

Figure 3. Time dependence of the quasiparticle number in the volume $\lambda^{3}$ in the weak periodic field with the parameters $E / E_{c r}=2 \cdot 10^{-5}$ and $\lambda=800 \mathrm{~nm}$, corresponding to the Jena Ti:sapphire laser ${ }^{28}$ (solid line) and in the nearcritical field case of an X-ray laser ${ }^{32}$ with $E / E_{c r}=0.24$ and $\lambda=0.15 \mathrm{~nm}$ (dashed line).

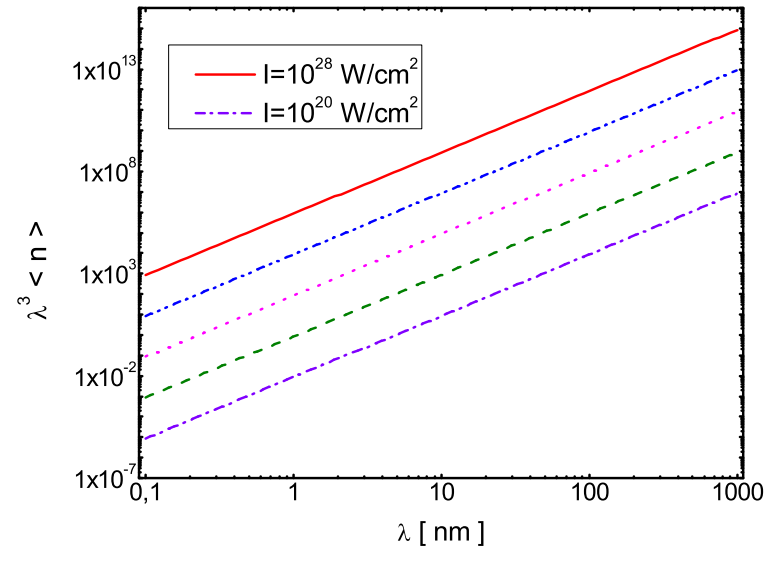

Figure 4. $\lambda$-dependence of the mean quasiparticle number in the volume $\lambda^{3}$ for different field strengths. The lines are drawn with a step width of $10^{2} \mathrm{~W} / \mathrm{cm}^{2}$. The mean number density of pairs $\langle n\rangle$ is independent of the wave length in this range of parameters.

Most of the previous works dealing with the estimation of pair production in a laser field (e.g. ${ }^{9,11}$ ) have considered the particles which remain after switching off the external field (residual or true particles). In this setting, the obtained results were negative: the residual pair density is not observable for field strengths $E \ll E_{c r}=m^{2} / e$. The other possibility was investigated in Ref. ${ }^{14}$ The dynamical pair density during the action of laser pulse changes periodically with twice the field frequency and its mean value exceeds the residual density by many orders of magnitude, see Fig. 3. We suppose that these "quasi-particles" can undergo the standard processes as, e.g., two-photon annihilation providing an indirect channel for the observation of the dynamical Schwinger effect.

\section{OBSERVABLE EFFECTS}

The peculiarity of the considered phenomenon is that the created pairs are existing during the laser pulse only and decay almost completely after the disappearance of the beams. Nevertheless, in comparison with XFELs, modern optical lasers can generate more pairs owing to the larger spot volume, and hence may provide now access to observable signals of vacuum decay in indirect ways, such as the production of coincident photon pairs from $e^{+} e^{-}$annihilation (Fig. 1) or the change of the refraction index (Fig. 2).

The estimations made in Ref. ${ }^{14}$ in the framework of the S-matrix formalism show that the operating lasers can produce in one shot a few tens of $\gamma$-quanta, which can be detected by a coincidence counter. The rate of two-photon annihilation grows as $E^{4}$, therefore the planned increase of laser intensity to $10^{24}-10^{25} \mathrm{~W} / \mathrm{cm}^{3}$ will result in a huge increase of the $2 \gamma$ flux. A further increase of the intensity to $10^{26}-10^{28} \mathrm{~W} / \mathrm{cm}^{3}$ opens the channels for creation of heavier particles such as charged muon and pion pairs ${ }^{15}$ which annihilate into still harder $\gamma$-quanta with energies in the range of $100-200 \mathrm{MeV}$.

Another experimental method to reveal the $e^{+} e^{-}$-plasma is to use an additional probe laser for measuring the plasma refraction index, see Fig. 2. The collision of two counter-propagating laser beams with wavelength $\lambda_{0}$ forms in the focus a standing wave with thin regions ("pancakes") filled by an electron-positron plasma with pulsing density $n(t) \sim \sin ^{2}\left(\omega_{0} t\right)$. The direction of the probe laser beam can be chosen in the range $0-90^{\circ}$ which influences insignificantly on the effective path length of beam in the presence of the plasma under typical conditions of high-power lasers where the waist of focus is a few wave length and the pulse duration is $10-100$ 


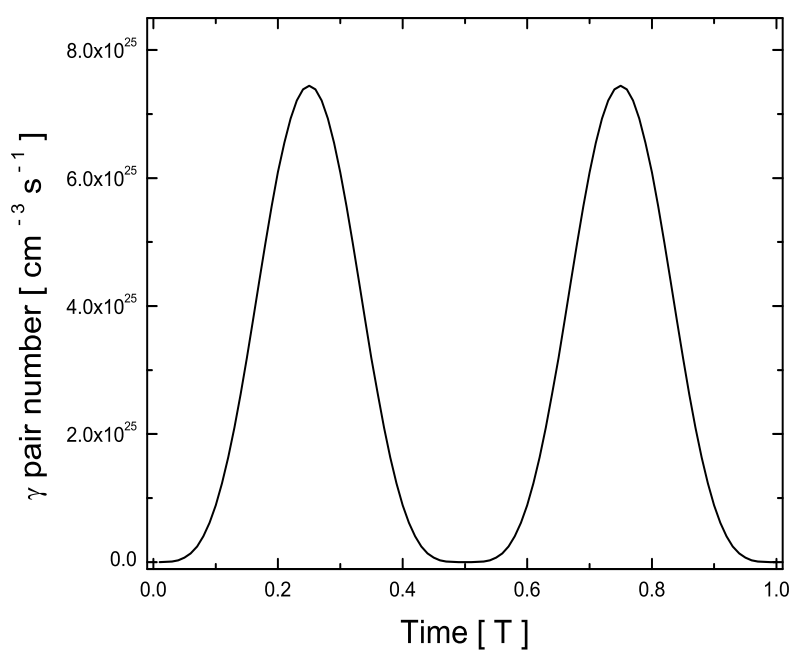

Figure 5. The time dependence of annihilation rate (12) of $e^{+}-e^{-}$pairs in $\lambda_{0}^{3}$ volume for parameters of Jena laser: ${ }^{28} \mathrm{I}=10^{20} \mathrm{~W} / \mathrm{cm}^{2}, \lambda_{0}=800 \mathrm{~nm}$.

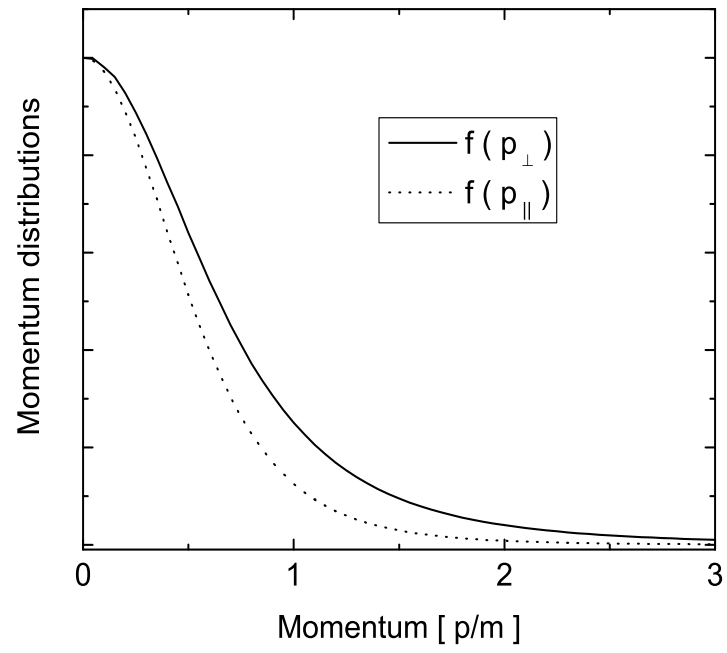

Figure 6. The momentum dependence of created $e^{+}-e^{-}$ pairs.

periods. We consider the beam of a probe laser with short wavelength $\lambda_{p} \ll \lambda_{0}$ propagating perpendicular to the direction of the high-power laser beams.

According Fig. 66, the momentum spectrum of created pairs is well modeled by a thermal equilibrium distribution with $T \sim m$ in the momentum range $p \lesssim m$. Assuming also that the probe pulse duration is short enough $\tau_{p} \ll \tau_{0}$, we can estimate the refraction index in the quasi-stationary and high frequency approximations, respectively. The problem reduces to a well-known one: the propagation of transverse waves in an equilibrium plasma with a Debye length $r_{D} \sim 10^{-5} \mathrm{~cm}<\lambda_{0}$ and a Langmuir frequency $\omega_{L} \sim 10^{14} / \mathrm{c}$.

In the high frequency approximation $\omega_{p} \gg k v_{T}$, the real part of the transverse dielectric permeability ${ }^{33}$ is

$$
\varepsilon_{t r}=1-\frac{\omega_{L}^{2}}{\omega_{p}^{2}}\left[1+\left(\frac{k v_{T}}{\omega_{p}}\right)^{2}\right] .
$$

The solution of the dispersion equation of transverse waves can be written as

$$
\omega_{p}^{2}=\omega_{L}^{2}+k^{2}\left[1+\frac{\omega_{L}^{2}}{\omega_{L}^{2}+k^{2}}\right]
$$

and the refraction index is

$$
n=\sqrt{\frac{1-\eta^{2}}{1+\eta^{2}}},
$$

where $\eta=\omega_{L} / \omega_{p}$. It follows that $n=0.999975$ for $\omega_{p}=10 \omega_{0}$ and $n=0.99999975$ for $\omega_{p}=100 \omega_{0}$. Such small differences of refraction indices of the split probe beams appears to be a challenge for the experimental detection by an interference pattern because of the rather short path in the plasma. To comfortably detectable, the value of the refraction index of the plasma should increase by about two orders of magnitude so that a clear interference pattern emerges. The increase of the laser intensity to $\mathrm{I}=10^{22}-10^{23} \mathrm{~W} / \mathrm{cm}^{2}$ will apparently be sufficient to create the conditions for observability of an interference pattern for the high-frequency probe beams. The more comprehensive calculation of the refraction index is performed in the work. ${ }^{34}$

The registration of higher harmonics is one more perspective method for the experimental observation of non-linear QED effects in a strong laser field. Here we investigate the spectrum of oscillations of the backreaction field (7). Fig. 7 7 shows that the third harmonics of the laser frequency $\omega_{0}$ is the most intensive one and its amplitude increases together with the laser wave length. The expected value of the amplitude for the third harmonics reaches the order of $10^{6} \mathrm{~V} / \mathrm{cm}$ for a laser intensity of $10^{20} \mathrm{~W} / \mathrm{cm}^{2}$. 

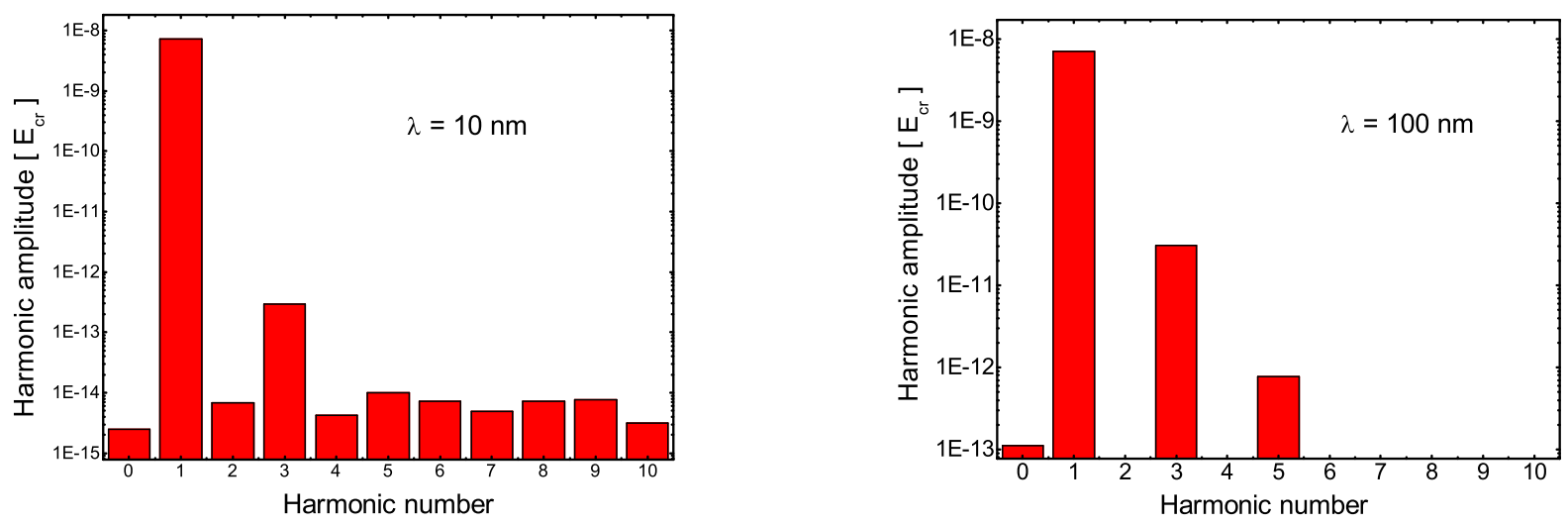

Figure 7. The dependence of the amplitudes of higher harmonics in the back-reaction field on the harmonics number.

\section{CONCLUSION}

Our estimates show that in the field of optical lasers with an intensity of $10^{28} \mathrm{~W} / \mathrm{cm}^{2}$ one can expect $\sim 10^{16}$ $e^{+}-e^{-}$annihilation events per laser pulse accompanied by irradiation of $\sim 1 \mathrm{MeV} \gamma$-quanta which can be registered outside the focus of the counter-propagating laser beams by coincidence counters. The expected amplitude of the third harmonics of the laser frequency reaches the order of $10^{6} \mathrm{~V} / \mathrm{cm}$ at a laser intensity of $10^{20}$ $\mathrm{W} / \mathrm{cm}^{2}$. The interference scheme for the observation of a refraction index of the transient $e^{+}-e^{-}$plasma can become feasible by a further increase of the laser intensity up to $10^{23}-10^{24} \mathrm{~W} / \mathrm{cm}^{2}$.

\section{REFERENCES}

1. G. Mourou, T. Tajima and S. V. Bulanov, "Optics in the relativistic regime," Rev. Mod. Phys. 78, p. 309, 2006.

2. S. Bulanov, T. Esirkepov, and T. Tajima, "Light Intensification towards the Schwinger Limit," Phys. Rev. Lett. 91, p. 085001, 2003.

3. F. Sauter, "Über das Verhalten eines Electrons im homogenen elektrischen Feld nach der relativistischen Theorie Diracs," Z. Phys. 69, p. 742, 1931.

4. W. Heisenberg and H. Euler, "Consequences of Dirac's Theory of Positrons," Z. Phys. 98, p. 714, 1936.

5. J. Schwinger, "On gauge invariance and vacuum polarization," Phys. Rev. 82, pp. 664-679, 1951.

6. C. Troup and H. Perlman, "Pair production in a vacuum by an alternating field," Phys. Rev. D 6, p. 2299, 1972 .

7. M. Marinov and V. Popov, "Electron-positron pair creation from vacuum induced by variable electric field," Fortschr. Phys. 25, p. 373, 1977.

8. B. Richards and E. Wolf Proc. Roy. Soc. A (London) 253, p. 358, 1950.

9. F. Bunkin and I. Tugov, "On possibility of electron-positron pairs creation in vacuum by laser radiation focusing," Sov. Phys. Dokl. 14, pp. 678-681, 1969.

10. E. Brezin and C. Itzykson, "Pair production in vacuum by an alternating field," Phys. Rev. D 2, pp. 11911199, 1970.

11. V. Popov, "On Schwinger mechanism of $\mathrm{e}^{+} \mathrm{e}^{-}$pair production from vacuum by the field of optical and $\mathrm{x}$-ray lasers," Phys. Lett. A 298, pp. 83-90, 2002.

12. S. Bulanov, A. Fedorov, and F. Pegoraro, "Damping of electromagnetic waves due to electron-positron pair production," Phys. Rev. E 71, p. 016404, 2005. 
13. A. V. Prozorkevich, A. Reichel, S. A. Smolyansky, and A. V. Tarakanov, "The pair creation in the optical laser field," in 'Saratov Fall Meeting 2003: Laser Physics and Photonics, Spectroscopy, and Molecular Modeling IV', Proceedings of SPIE, v. 5476, pp. 68-72, SPIE, (Washington, USA), 2004.

14. D. B. Blaschke, A. V. Prozorkevich, C. D. Roberts, S. M. Schmidt, and S. A. Smolyansky, "Pair production and optical lasers," Phys. Rev. Lett. 96, p. 140042, 2006.

15. D. Blaschke, A. Prozorkevich, S. Smolyansky, and A. Tarakanov, "Observable manifestation of an electronpositron plasma created by the field of an optical laser," Journal of Physics: Conference Series 35, pp. 121$126,2006$.

16. A. Filatov, A. Prozorkevich, and S. Smolyansky, "Pair creation by electromagnetic wave in a collisionless plasma," in 'Saratov Fall Meeting 2005: Laser Physics and Photonics, Spectroscopy, and Molecular Modeling VI', Proceedings of SPIE, v. 6165, p. 616509, SPIE, (Washington, USA), 2006.

17. A. Tarakanov, N. Tarakanov, A. Prozorkevich, and S. Smolyansky, "Electron-positron pair creation by a multimode field of the optical laser," in 'Saratov Fall Meeting 2005: Laser Physics and Photonics, Spectroscopy, and Molecular Modeling VI', Proceedings of SPIE, v. 6165, p. 61650a, SPIE, (Washington, USA), 2006.

18. E. Esarey, S. Ride, and P. Sprangle, "Nonlinear Thomson scattering of intense laser pulses from beams and plasmas," Phys. Rev. E 48, pp. 3003-3021, 1993.

19. L. Landau and E. Lifshitz, Quantum Electrodynamics, Volume 4 (Course of Theoretical Physics Series), Butterworth-Heinemann; 2 ed., 1982.

20. D. Ivanov, G. Kotkin, and V. Serbo, "Complete description of polarization effects in $\mathrm{e}^{+} \mathrm{e}^{-}$pair production by a photon in the field of a strong laser wave," Eur. Phys. J. C 40, pp. 27-40, 2005.

21. T. Heinzl, B. Liesfeld, K.-U. Amthor, H. Schwoerer, R. Sauerbrey, and A. Wipf, "On the observation of vacuum birefringence," Opt. Commun. 267, pp. 318-321, 2006.

22. J. S. Heyl and L. Hernquist, "Birefringence and Dichroism of the QED Vacuum," J. Phys. A 30, p. 6485, 1997.

23. A. D. Piazza, K. Hatsagortsyan, and C. Keitel, "Enhancement of vacuum polarization effects in a plasma," hep-ph/0606059.

24. D. Kharzeev and K. Tuchin, "Vacuum self-focussing of very intense laser beams," Phys. Rev. A 75, 043807 (2007) arXiv:hep-ph/0611133.

25. N. Narozhny and A. Nikishov, "The simplest processes in the pair creating electric field," Sov. J. Nucl. Phys. 11, p. 596, 1970.

26. A. Grib, S. Mamaev, and V. Mostepanenko, Vacuum quantum effects in strong external fields, Friedman Publisher company, St. Petersburg, 1991.

27. S. Schmidt, D. Blaschke, G. Ropke, S. Smolyansky, A. Prozorkevich, and V. Toneev, "A quantum kinetic equation for particle production in the Schwinger mechanism," Int. J. Mod. Phys. E 7, pp. 709-722, 1998.

28. B. Liesfeld, J. Bernhardt, K.-U. Amthor, H. Schwoerer, and R. Sauerbrey, "Single-shot autocorrelation at relativistic intensity," Appl. Phys. Lett. 86, p. 161107, 2005.

29. D. Vinnik, A. Prozorkevich, S. Smolyansky, V. Toneev, M. Hecht, C. Roberts, and S. Schmidt, "Plasma production and thermalisation in a strong field," Eur. Phys. J. C 22, pp. 341-349, 2001.

30. R. Alkofer, M. B. Hecht, C. D. Roberts, S. M. Schmidt, and D. V. Vinnik, "Pair creation and an X-ray free electron laser," Phys. Rev. Lett. 87, p. 193902, 2001.

31. C. D. Roberts, S. M. Schmidt, and D. V. Vinnik, "Quantum effects with an X-ray free electron laser," Phys. Rev. Lett. 89, p. 153901, 2002.

32. A. Ringwald, "Pair production from vacuum at the focus of an X-ray free electron laser," Phys. Lett. B 510, p. 107, 2001.

33. L. Landau and E. Lifshitz, Physical Kinetics, Volume 10 (Course of Theoretical Physics Series), Butterworth-Heinemann; 1 ed., 1981.

34. D. B. Blaschke, A. V. Prozorkevich, G. Roepke, C. D. Roberts, S. M. Schmidt, D. S. Shkirmanov and S. A. Smolyansky, "Dynamical Schwinger effect and high-intensity lasers. Realising nonperturbative QED," arXiv:0811.3570 [physics.plasm-ph]. 